\title{
Construction of Orthogonal Basis Functions with Non-Divergent Barotropic Rossby-Haurwitz Waves
}

\author{
Hyeong-Bin Cheong*, Hanbyeol Jeong, and Wonho Kim \\ Department of Environmental Atmospheric Sciences, Pukyong National University, \\ Busan 608-737, Korea
}

\begin{abstract}
A new set of basis functions was constructed using the Rossby-Haurwitz waves, which are the eigenfunctions of nondivergent barotropic vorticity equations on the sphere. The basis functions were designed to be non-separable, that is, not factored into functions of either the longitude or the latitude. Due to this property, the nodal lines of the functions are aligned neither along with the meridian nor the parallel. The basis functions can be categorized into groups of which members have the same degree or the total wavenumber-like index on the sphere. The orthonormality of the basis functions were found to be close to the machine roundoffs, giving the error of $O\left(10^{-15}\right)$ or $O\left(10^{-16}\right)$ for double-precision computation (64 bit arithmetic). It was demonstrated through time-stepping procedure that the basis functions were also the eigenfunctions of the non-divergent barotropic vorticity equations. The projection of the basis functions was carried out onto the low-resolution geopotential field of Gaussian bell, and compared with the theory. The same projections were performed for the observed atmospheric-geopotential height field of $500 \mathrm{hPa}$ surface to demonstrate decomposition into the fields that contain disturbance of certain range of horizontal scales. The usefulness of the new basis functions was thus addressed for application to the eigenmode analysis of the atmospheric motions on the global domain.
\end{abstract}

Keywords: Rossby-Haurwitz waves, geopotential, basis function, spherical harmonic functions, eigenmodes, orthogonality

\section{Introduction}

Geophysical phenomena on the spherical surface are in general described by differential equations expressed with the spherical coordinates system (Swarztrauber, 1993, 1996; Holton, 2004; Flyer et al., 2012). Geophysical gridded-data commonly adopts lat-lon grid system where the grids are aligned along the spherical coordinate axes. This gives many advantages when dealing with a discrete analogue of the differential equations governing geophysical phenomena. Among them, the most important one may be the structuredness of the grid, which allows the use of efficient data structure on digital computers. For instance, the gridded-data covering the whole sphere can be

\footnotetext{
*Corresponding author: hbcheong@pknu.ac.kr Tel: +82-51-629-6642

Fax: $+82-51-629-6636$
}

This is an Open-Access article distributed under the terms of the Creative Commons Attribution Non-Commercial License (http:// creativecommons.org/licenses/by-nc/3.0) which permits unrestricted non-commercial use, distribution, and reproduction in any medium, provided the original work is properly cited. represented as two dimensional arrays.

Since the global data are periodic in the longitudinal direction on the spherical coordinate system, the Fourier transform can be conveniently used to represent the data in terms of the wave space (Swarztrauber, 1993; Jekeli et al., 2007; Cheong et al., 2012). In particular, this periodicity can be more important in the linearized system, e.g., a shallow water system, because they can be transformed into only latitudedependent differential equations through Fourier analysis. This makes the dynamical analysis much simpler and easier. However, the use of these zonally Fourier-transformed equations implies that their solution yield only separable functions; therefore some important features are unlikely to be captured appropriately by this approach.

Recently, the global grid systems, which are not of lat-lon structure, have emerged in an attempt to provide a nearly uniform resolution over the globe and to avoid the non-uniformity of the lat-lon grid system (Heikes and Randall, 1995; Stuhne and Peltier, 1995; Nair, 2009; Flyer et al., 2012; Heikes et al., 2013). There are many uniform grid systems, as illustrated by 
Flyer et al. (2012) and Weller (2012). Icosahedral grids and the cubed-sphere grids are considered two representative almost-uniform grids, with which global numerical models have been developed over the past two decades. A notable feature of these grid systems is that the eigensolution of dynamical systems discretized on these grids is fundamentally different from those obtained from traditional lat-lon grids (Thuburn et al., 2009; Weller, 2012). Thuburn et al. (2009) have found that the eigenfunctions of the spherical Laplacian operator is not separable into either the longitude or the latitude. Most of the eigenfunctions reflected the grid structures: The cubed sphere grid was found to have eigenfunctions with a 6-fold symmetry, while the icosahedral grid was shown to provide 12 - or 20 -fold symmetric structures. These results imply that the eigensolutions of other linear systems may exhibit non lat-lon structure. Although the non lat-lon grids such as the icosahedral- and cubed sphere-grid provide nonseparable eigenfunctions, it is not feasible to express them in terms of analytical formula. Therefore, the application of the non-separable eigensolutions to the analysis of geophysical problems including the atmospheric dynamics may be quite limited. The purpose of this paper is hence to construct nonseparable basis functions which can be expressed in analytical formula.

This paper is organized as follows. In the next section, the nondivergent barotropic equations on the sphere and associated solutions are briefly described. Then, a new set of orthonormal basis functions are constructed using the Legendre functions or the Rossby-Haurwitz waves, and orthonormality of the functions are evaluated in the following section. In the final section, summary and conclusion is presented.

\section{Non-divergent Barotropic Rossby-Haurwitz Waves and Legendre Functions}

The non-divergent barotropic vorticity equation on a unit sphere is written as (Dickinson, 1978; Holton, 2004; Cheong and Park, 2007):

$$
\frac{\partial \zeta}{\partial t}=-\frac{u}{\cos \theta} \frac{\partial}{\partial \lambda}(\zeta+f)-v \frac{\partial}{\partial \theta}(\zeta+f)
$$

where $\lambda$ and $\theta$ are the longitude and the latitude, respectively, and $u$ and $v$ denote the nondivergent, longitudinal- and latitudinal-velocity component, respectively, $\zeta$ is the vorticity, and $[=2 \Omega \sin \theta]$ means the Coriolis parameter with $\Omega$ being the Earth's rotation rate. Introducing the streamfunction, the vorticity and the velocities are expressed as diagnostic equations as

$$
\begin{aligned}
& \zeta=\frac{1}{\cos ^{2} \theta}\left[\frac{\partial^{2} \psi}{\partial \lambda^{2}}+\cos \theta \frac{\partial}{\partial \theta} \cos \theta \frac{\partial \psi}{\partial \theta}\right] \\
& u=-\frac{\partial \psi}{\partial \theta} \\
& v=\frac{1}{\cos \theta} \frac{\partial \psi}{\partial \lambda} .
\end{aligned}
$$

The linearized form of (1) for the super-rotating atmosphere is written in terms of the streamfunction as

$\frac{\partial}{\partial t} \nabla \psi=-2 \Omega \frac{\partial \psi}{\partial \lambda}$

The streamfunction, which is one of the square integrable scalar functions, can be expanded with infinite series of the Legendre functions:

$$
\begin{aligned}
& \psi(\lambda, \theta, t)=\operatorname{Re}\left[\sum_{m=-\infty}^{\infty} \sum_{n=|m|}^{\infty} \hat{\psi}_{n, m}(t) P_{n}^{|m|}(x) e^{i m \lambda}\right], \\
& i=\sqrt{-1}
\end{aligned}
$$

where $x=\sin \theta, m$ is the zonal wavenumber, $P_{n}^{|m|}$ implies the Legendre function with the order $m$ and degree $n$, and $\psi_{n, m}(t)$ represents the spectral coefficient of complex variable. The spherical Laplacian of the streamfunction satisfies the following identity, because the Legendre functions are the eigensolutions of the spherical Laplacian operator as:

$$
\frac{d}{d x}\left(1-x^{2}\right) \frac{d}{d x} P_{n}^{m}(x)-m^{2} \frac{P_{n}^{m}}{1-x^{2}}+[n(n+1)] P_{n}^{m}(x)=0 .
$$

On the whole domain on the spherical surface, the 
Legendre polynomials constitute the orthonormal basis such that

$$
\int_{-1}^{+1} P_{n}^{m}(x) P_{l}^{m}(x) d x=\delta_{n, l}
$$

where $\delta_{n, l}$ means Kronecker delta. Substituting (4) into (3), an ordinary differential equation for the spectral coefficients is obtained as (Rossby, 1939; Aleksey et al., 2003):

$$
\frac{d}{d t} \hat{\psi}_{n, m}=\frac{2 i \Omega m}{n(n+1)} \psi_{n, m},
$$

With the function as $\hat{\psi}_{n, m}=\Psi e^{i w t}$, (7) yields the dispersion relation of the Rossby-Haurwitz wave:

$$
w=2 \Omega \frac{m}{n(n+1)},
$$

which gives the phase speed of westward propagation (Boyd, 1980; Holton, 2004; Cai and Huang, 2013):

$$
c_{p} \equiv \frac{w}{m}=\frac{2 \Omega}{n(n+1)} .
$$

Orthonormal basis using Rossby-Haurwitz waves (Legendre functions)

Using the orthonormality of the Rossby-Haurwitz waves (Legendre functions with $n>0$ ), new set of orthonormal basis functions, which are not separable in the longitude and the latitude, can be constructed:

$$
\begin{aligned}
& Q_{n, k}(\lambda, \theta)=\frac{1}{\sqrt{4 \pi}} a_{n, k, 0} P_{n}^{0}(x), \\
& +\frac{1}{\sqrt{2 \pi}} \sum_{m=1}^{n}\left[a_{n, k,-m} \cos m \lambda+a_{n, k, m} \sin m \lambda\right] P_{n}^{m}(x)
\end{aligned}
$$

where $k$ is the mode number, and $a_{n, k, m}$ represents the real-valued coefficients of the Spherical Harmonic Functions (Dilts, 1985; Moriguchi et al., 1990; Nehrkorn, 1990; Swarztrauber, 1993; Sneeuw and Bun, 1996; Jekeli et al., 2007; Cheong et al., 2012). The function in (9) includes more than one zonalwavenumber component, hence, of which structure is not any more separable in the longitude and the latitude. As the degree becomes higher, more zonal- wavenumber components are included in the basis function. One important property that is required for the basis functions is the orthonormality:

$$
\int_{0}^{2 \pi} \int_{-1}^{1} Q_{n^{\prime}, k^{\prime}} Q_{n, k} d x d \lambda=\delta_{n, n^{\prime}} \delta_{k, k^{\prime}}
$$

From the orthonormality of the SHFs, which are used as building blocks of the new orthogonal basis functions, the requirement of (11) can be expressed in terms of $a_{n, k, m}$ as

$$
\sum_{m=-n}^{n} a_{n, k, m} a_{n, k^{\prime}, m}=\delta_{k, k^{\prime}} .
$$

The Fourier functions, by virtue of the orthogonality, can be an appropriate candidate for $a_{n, k, m}$ :

$$
\begin{aligned}
& a_{n, k, m}=\frac{c}{\sqrt{2 n+1}} \sin \left[\frac{(k+n+1)(m+n+0.5) \pi}{2 n+1}\right], \\
& c= \begin{cases}1 & \text { for } k=n \\
2 & \text { for } k \neq n\end{cases}
\end{aligned}
$$

where $(k+n+1)$ plays a role as the wavenumber and $(m+n+0.5)$ plays sampling point-like role (data points) in the Fourier analysis and synthesis. The function, that characterizes the expansion coefficients $a_{n, k, m}$, can be determined differently with the degree $n$. If $a_{n, k, m}$, i.e., the coefficients of the SHFs, is plotted as a function of $(m+n+0.5)$, it will look like the sine functions with different wavenumber.

Differentiation of $Q_{n, k}(\lambda, x)$ with respect to the longitude and the latitude is straightforward, because their structures are given in terms of the SHFs:

$$
\begin{aligned}
& \frac{\partial}{\partial \lambda} Q_{n, k}(\lambda, \theta) \\
& +\frac{1}{\sqrt{2 \pi}} \sum_{m=1}^{n}\left[a_{n, k, m} \cos m \lambda-a_{n, k,-m} \sin m \lambda\right] m P_{n}^{m}(x)
\end{aligned}
$$

and

$$
\begin{aligned}
& \cos \theta \frac{\partial}{\partial \theta} Q_{n, k} \\
& =\left(1-x^{2}\right) \frac{\partial}{\partial x} Q_{n, k}
\end{aligned}
$$




$$
\begin{aligned}
& =\frac{1}{\sqrt{4 \pi}} a_{n, k, 0}\left[-n \varepsilon_{n+1,0} P_{n+1}^{0}+(n+1) \varepsilon_{n, 0} P_{n-1}^{0}\right] \\
& +\frac{1}{\sqrt{2 \pi}} \sum_{m=1}^{n} a_{n, k,-m} \cos m \lambda\left[-n \varepsilon_{n+1, m} P_{n+1}^{m}+(n+1) \varepsilon_{n, m} P_{n-1}^{m}\right] \\
& +\frac{1}{\sqrt{2 \pi}} \sum_{m=1}^{n} a_{n, k, m} \sin m \lambda\left[-n \varepsilon_{n+1, m} P_{n+1}^{m}+(n+1) \varepsilon_{n, m} P_{n-1}^{m}\right]
\end{aligned}
$$

where

$$
\varepsilon_{n, m}=\sqrt{\frac{n^{2}-m^{2}}{4 n^{2}-1}} .
$$

Projection of the basis functions to their differentiation can be performed through the orthogonality of the Fourier series and Legendre functions (Nehrkorn, 1990):

$$
\begin{aligned}
& \int_{0}^{2 \pi} \int_{-1}^{1} Q_{n, k}\left[\frac{\partial}{\partial \lambda} Q_{n^{\prime}, k^{\prime}}\right] d x d \lambda \\
& =\sum_{m=-n}^{n}\left[a_{n, k,-m} a_{n, k^{\prime}, m}-a_{n, k, m} a_{n, k^{\prime},-m}\right]
\end{aligned}
$$

and

$$
\begin{aligned}
& \int_{0}^{2 \pi} \int_{-1}^{1} Q_{n, k}\left(1-x^{2}\right)\left[\frac{\partial}{\partial \lambda} Q_{n^{\prime}, k^{\prime}}\right] d x d \lambda \\
& =\frac{1}{\sqrt{4 \pi}} \sum_{m=1}^{n} c_{m}\left[(n+2) \varepsilon_{n+1, m} a_{n, k,-m} a_{n+1, k^{\prime},-m}\right] \\
& -\frac{1}{\sqrt{4 \pi}} \sum_{m=1}^{n} c_{m}\left[(n-1) \varepsilon_{n, m} a_{n, k, m} a_{n-1, k^{\prime}, m}\right] \\
& c=\left\{\begin{array}{c}
1 \text { for } m=0 \\
\sqrt{2} \text { for } m \neq 0
\end{array}\right.
\end{aligned}
$$

\section{Evaluation of the basis functions}

The orthonormality of the basis functions constructed in the previous section is evaluated. For numerical accuracy, the calculations are carried out with the double precision FORTRAN 90 compiler, where 16 digits of floating points are available. The basis functions are generated using the Legendre functions and the Fourier series on the Gaussian grids
(Nehrkorn, 1990; Swarztrauber, 1996). If not stated otherwise, the number of grids is given as $360 \times 180$ on the global domain. The Gaussian points, weights, and the Legendre functions are calculated by the method of Nehrkorn (1990), where two term recursion relations are incorporated. Table 1 shows the errors of orthogonality and normality: The normality error was presented as the value subtracted by unity, hence the smaller value implies higher accuracy. As can be seen in the Table, the errors are very close to machine roundoff, typically in the order of $O\left(10^{-15}\right)$ or $O\left(10^{-16}\right)$. This high accuracy seems to have come from the accuracy of the Gaussian quadrature used for the projection of the Legendre functions (Swarztrauber, 1996; Cheong et al., 2007). Some of the basis functions are presented in Fig. 1 for the degree $n=3$, where the orthographic projection was used for the longitudinal range of $[0, \pi]$. As stated above, the basis functions do not show nodal lines aligned along the meridian, indicating that they are not represented with separable functions (cf, Thuburn et al., 2009; Weller, 2012).

As stated above, the Rossby-Haurwitz waves of same degree propagate with a constant phase speed, regardless of the zonal wavenumber. Therefore, when Eq. (3) is used, the new basis functions are expected to propagate westward, with preserving the initial shape (Dickinson, 1978; Boyd, 1980; Cai and Huang, 2013). Time evolution of the basis function $Q_{3,2}(\lambda, x)$, whose frequency is $\Omega / 6$ is illustrated in Fig. 2, where time interval is a half day ( 6 days are required for one complete revolution). It is clear that the initial pattern moves westward with a constant speed without changing the spatial pattern with time. This means that the basis function is also the normal mode of Eq. (7), the linear nondivergent barotropic equation.

Any gridpoint data defined on Gaussian grids can be decomposed into the new basis functions due to their orthonormality. To demonstrate this, a lowresolution global data with $n \leq 12$, given as a Gaussian bell type, was expanded with the new basis functions.

$$
y\left(\theta^{\prime}\right)=\exp \left[-a \sin ^{2}\left(\theta^{\prime} / 2\right)\right]
$$


Table 1. The errors of the normality and orthogonality for the non-separable basis functions

\begin{tabular}{|c|c|c|c|c|c|c|c|}
\hline$n$ & $k$ & $\gamma_{1}$ & $\gamma_{2}$ & $n$ & $k$ & $\gamma_{1}$ & $\gamma_{2}$ \\
\hline 0 & 0 & $-0.31 \mathrm{E}-16$ & $-0.34 \mathrm{E}-16$ & 4 & 2 & $-0.35 \mathrm{E}-16$ & $-0.22 \mathrm{E}-15$ \\
\hline 1 & 0 & $+0.33 \mathrm{E}-16$ & $-0.12 \mathrm{E}-15$ & 4 & 3 & $-0.43 \mathrm{E}-16$ & $-0.13 \mathrm{E}-15$ \\
\hline 1 & 1 & $-0.43 \mathrm{E}-16$ & $+0.56 \mathrm{E}-16$ & 4 & 4 & $+0.56 \mathrm{E}-16$ & $+0.64 \mathrm{E}-16$ \\
\hline 1 & 2 & $-0.13 \mathrm{E}-15$ & $-0.20 \mathrm{E}-15$ & 4 & 5 & $-0.17 \mathrm{E}-15$ & $-0.17 \mathrm{E}-15$ \\
\hline 2 & 0 & $-0.23 \mathrm{E}-16$ & $-0.38 \mathrm{E}-16$ & 4 & 6 & $+0.28 \mathrm{E}-16$ & $+0.32 \mathrm{E}-16$ \\
\hline 2 & 1 & $+0.17 \mathrm{E}-15$ & $+0.11 \mathrm{E}-15$ & 4 & 7 & $-0.29 \mathrm{E}-16$ & $-0.16 \mathrm{E}-15$ \\
\hline 2 & 2 & $-0.53 \mathrm{E}-16$ & $+0.66 \mathrm{E}-16$ & 4 & 8 & $-0.22 \mathrm{E}-15$ & $-0.21 \mathrm{E}-16$ \\
\hline 2 & 3 & $-0.23 \mathrm{E}-16$ & $+0.12 \mathrm{E}-16$ & 5 & 0 & $+0.19 \mathrm{E}-16$ & $+0.99 \mathrm{E}-16$ \\
\hline 2 & 4 & $+0.72 \mathrm{E}-16$ & $-0.94 \mathrm{E}-16$ & 5 & 1 & $-0.11 \mathrm{E}-15$ & $-0.30 \mathrm{E}-16$ \\
\hline 3 & 0 & $+0.15 \mathrm{E}-15$ & $+0.47 \mathrm{E}-16$ & 5 & 2 & $-0.26 \mathrm{E}-16$ & $-0.10 \mathrm{E}-15$ \\
\hline 3 & 1 & $-0.35 \mathrm{E}-16$ & $-0.17 \mathrm{E}-15$ & 5 & 3 & $+0.17 \mathrm{E}-16$ & $+0.64 \mathrm{E}-16$ \\
\hline 3 & 2 & $-0.22 \mathrm{E}-15$ & $-0.68 \mathrm{E}-16$ & 5 & 4 & $-0.12 \mathrm{E}-15$ & $-0.56 \mathrm{E}-16$ \\
\hline 3 & 3 & $+0.20 \mathrm{E}-16$ & $-0.37 \mathrm{E}-16$ & 5 & 5 & $+0.72 \mathrm{E}-16$ & $+0.13 \mathrm{E}-15$ \\
\hline 3 & 4 & $+0.11 \mathrm{E}-15$ & $-0.18 \mathrm{E}-15$ & 5 & 6 & $-0.97 \mathrm{E}-16$ & $-0.82 \mathrm{E}-16$ \\
\hline 3 & 5 & $+0.49 \mathrm{E}-16$ & $+0.29 \mathrm{E}-16$ & 5 & 7 & $-0.22 \mathrm{E}-15$ & $+0.87 \mathrm{E}-16$ \\
\hline 3 & 6 & $-0.21 \mathrm{E}-15$ & $-0.10 \mathrm{E}-16$ & 5 & 8 & $+0.23 \mathrm{E}-16$ & $-0.12 \mathrm{E}-15$ \\
\hline 4 & 0 & $-0.16 \mathrm{E}-16$ & $+0.09 \mathrm{E}-15$ & 5 & 9 & $-0.13 \mathrm{E}-16$ & $-0.47 \mathrm{E}-16$ \\
\hline 4 & 1 & $+0.17 \mathrm{E}-16$ & $-0.57 \mathrm{E}-16$ & 5 & 10 & $-0.13 \mathrm{E}-15$ & $-0.94 \mathrm{E}-16$ \\
\hline
\end{tabular}
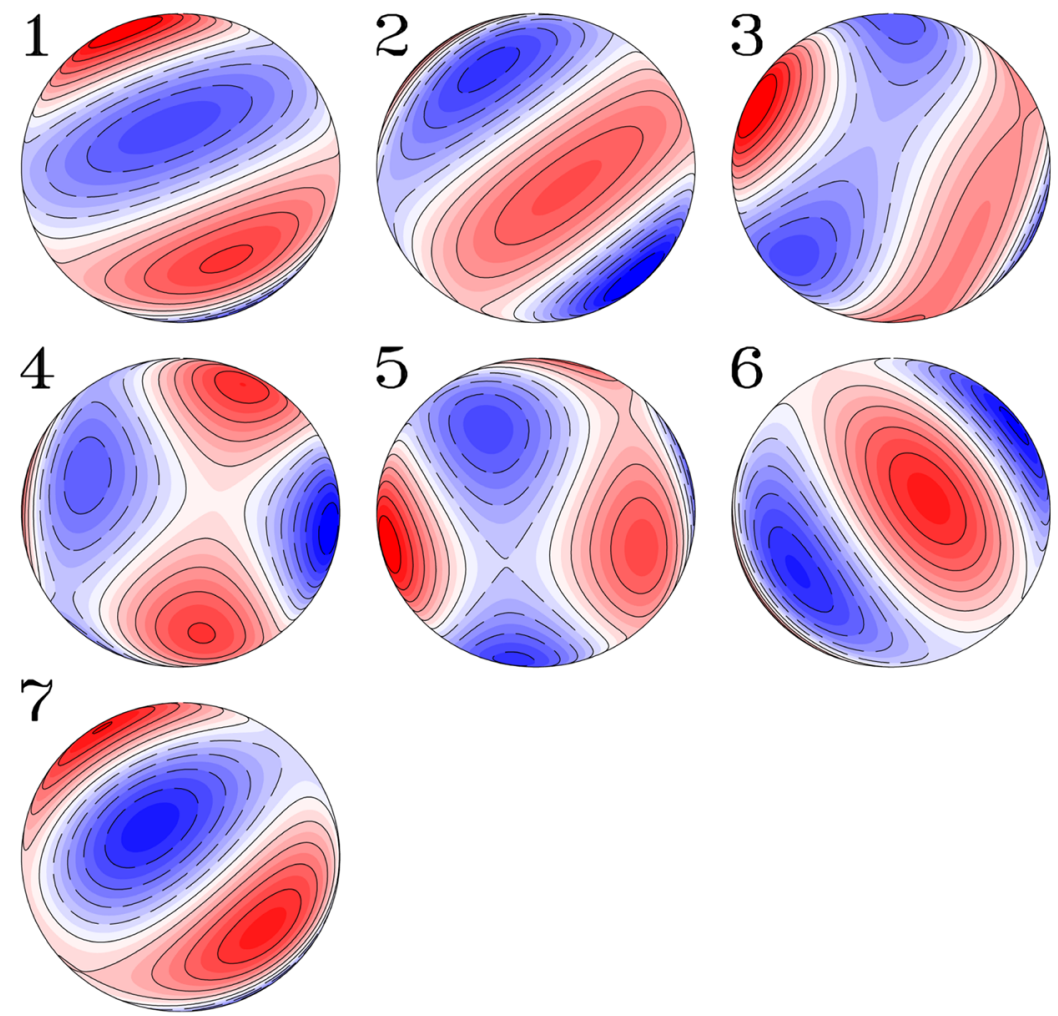

Fig. 1. New basis functions with the degree 3, which are represented on orthographic map projection. Numerals in the upper left corner means the mode number, and solid (dashed) lines are drawn for positive (negative) values with the contour interval of 0.1 . 

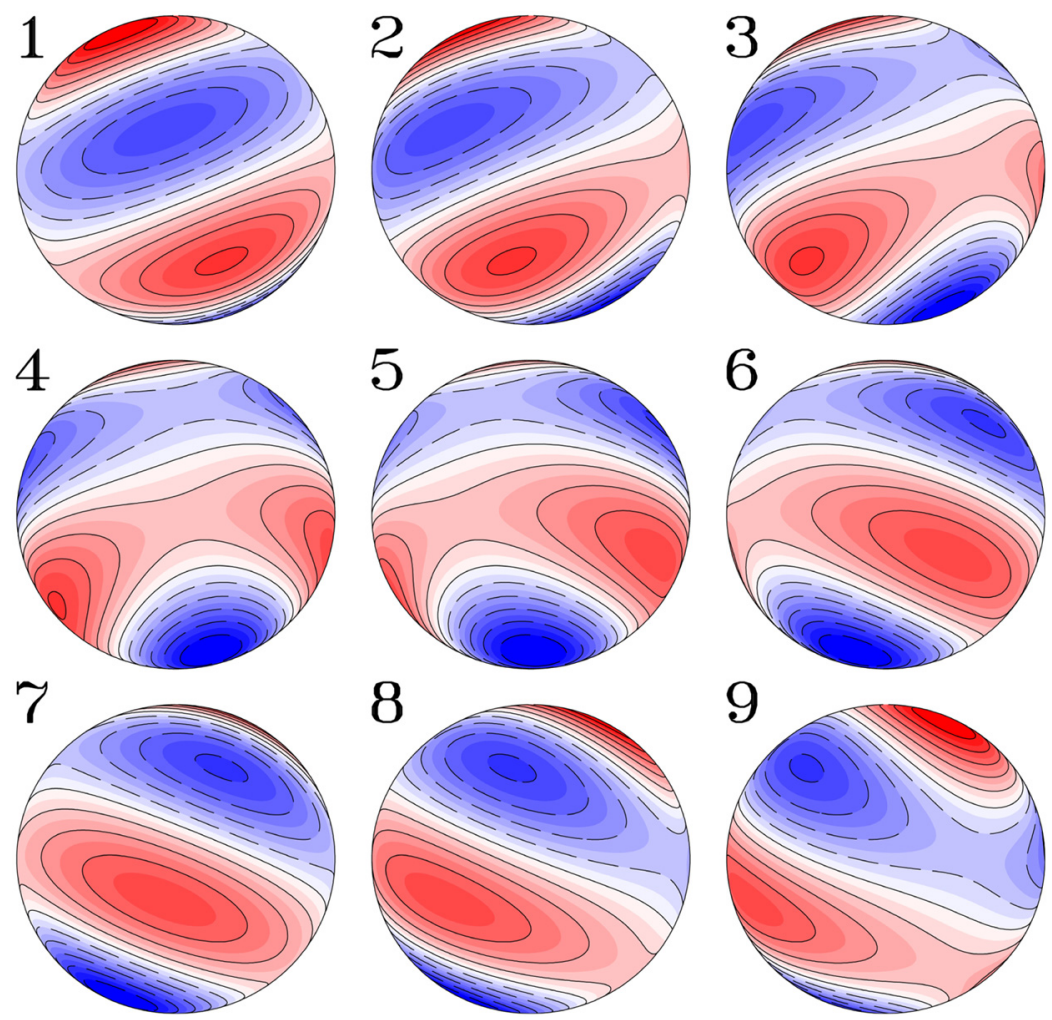

Fig. 2. Time sequence of the Rossby-Haurwitz waves consisting of the new basis function $Q_{3,2}(\lambda, x)$, of which frequency is $\Omega / 6$ with $\Omega$ the Earth's rotation rate. Wave patterns are presented in every 2 hours interval. Map projection and the contour interval are the same as in Fig. 1.
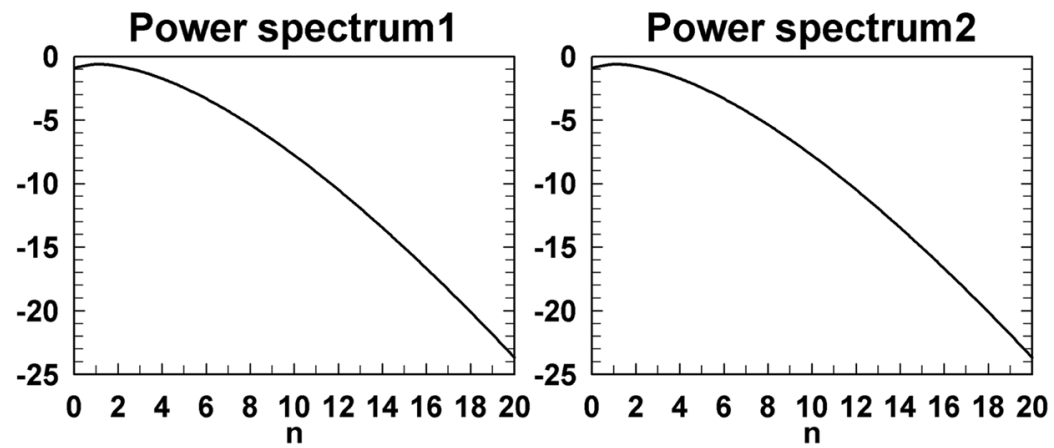

Fig. 3. Power spectrum in logarithmic scale (or degree variance) of a low-resolution global Gaussian-bell type geopotential field with the scale parameter of 10 . Left (right) panel represents the result from new basis functions (theory).

where $\theta^{\prime}$ means the angle from the center of Gaussian bell, and $a$ means the scale parameter which determines the horizontal scale of the Gaussian bell. Detailed procedure to calculate analytically the power spectrum can be found in Cheong and Kong (2013). The power spectrum (or degree variance) was presented in Fig. 3.
Two plots of power spectrum show no significant difference, both exhibiting exponential decay of the power with the horizontal scale (degree). The results in Fig. 3 suggest that the new basis functions are well capable of decomposing an arbitrary global field into discrete horizontal total wavenumber-like indices. 

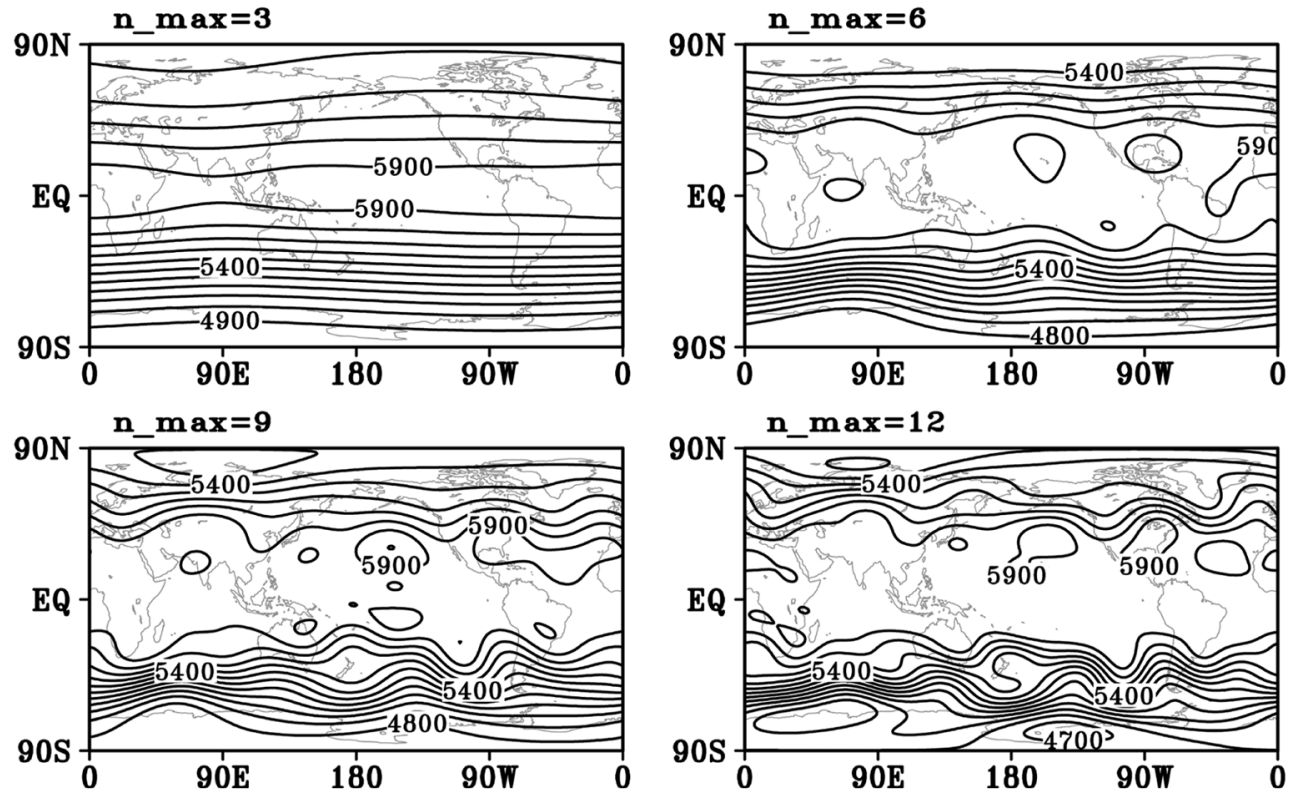

Fig. 4. Observed geopotential fields reconstructed with a certain range of spectral coefficient (i.e., horizontal scales) which was obtained by projecting the new basis functions to the geopotential height of $500 \mathrm{hPa}$ level at $1200 \mathrm{UTC} 31$ AUG 2012. Symbol n_max denotes the largest wavenumber contained in each global field.

Application to real observed atmospheric geopotential data is illustrated in Fig. 4: Observed data of $500 \mathrm{hPa}$ level at 1200 UTC of 31 AUG 2010 is first projected onto the new basis functions, and then the global gridpoint data are reconstructed using the spectral coefficient for a certain range of the degree. Four maps presented in this figure are for $n \leq 3, n \leq 6, n \leq 9$, and $n \leq 12$. The fields become more wavy as the range of the coefficient becomes large, that is, the field is composed of ultralong scales for $n \leq 3$ while it contains more smaller horizontal scales for $n \leq 12$. This example also demonstrates the usefulness of the new basis functions in decomposing the global data into the fields of the same degree.

\section{Summary and Conclusion}

In this study, a new set of basis functions have been constructed based on the Rossby-Haurwitz waves, which are the normal modes of nondivergent barotropic vorticity equations on the sphere. The most significant feature of the new basis functions is that they are not separable, implying that they do not consist of a single zonal wavenumber. In other words, they cannot be decomposed into functions of the longitude or the latitude. The basis functions can be categorized into groups which have the same degree. Therefore, the basis functions of identical degree (in the same group) should have the same phase speed when they are time integrated with the linearized non-divergent barotropic vorticity equations.

Evaluation of the basis functions were carried out in terms of the orthonormality, and the errors were found to be about $O\left(10^{-15}\right)$ or $O\left(10^{-16}\right)$ for the computation with double precision (64 bit arithmetic). Time integration of the new basis functions demonstrated that the phase speed is the same as the Rossby-Haurwitz wave. The basis functions were projected on to a low-resolution geopotential field of Gaussian-bell type, and the power spectrum was shown to be well compared to the theory. Projection of the basis functions to the real observed meteorological data also demonstrated the capability of them to decompose the global fields into different horizontal scale or the degree. 
The new basis functions developed in this study can be applied to eigenmode analysis of the linear shallow water equations as well as the three dimensional equations governing the atmospheric motions. Such an analysis may be crucial to the detection of non lat-lon normal modes of the free atmospheric motions. The basis functions have a limitation in the aspect of computation because the projection of them requires $O\left(N^{4}\right)$ operation counts with the number of grids in zonal direction. This is much ineffective compared to the spherical harmonics basis which needs $O\left(N^{3}\right)$ operations. However, since the basis new functions retain the advantage of lat-lon grid system which is the only structured grid system on the global domain, the parallel computing could be achieved with efficiency and hence this limitation is not so serious. Such an extension to high-degree basis functions remains as future works.

\section{Acknowledgments}

This work was supported by a Research Grant of Pukyong National University (year 2014). Anonymous reviewers are acknowledged for careful reading of the manuscript and many useful comments.

\section{References}

Aleksey, V.Z., Szunyogh, I., Patil, D.J., Hunt, B.R., and Ott, E., 2003, Extracting envelopes of Rossby wave packets. Monthly Weather Review, 131, 1011-1017.

Boyd, J., 1980, Equatorial solitary waves. Part I: Rossby solitons. Journal of Physical Oceanography, 10, 16991717.

Cai, M. and Huang, B., 2013, A new look at the physics of Rossby waves: A mechanical-Coriolis oscillation. Journal of the Atmospheric Science, 70, 303-316.

Cheong, H.B. and Park, J.R., 2007, Geopotential field in nonlinear balance with the sectoral mode of RossbyHaurwitz wave on the inclined rotation axis. Journal of the Korean Earth Science Society, 28, 936-946.

Cheong, H.B., Park, J.R., and Kang, H.G., 2012, Fourierseries representation and projection of spherical harmonic functions. Journal of Geodesy, 86, 975-990.

Cheong, H.B. and Kong, H.J., 2013, Spherical harmonics power-spectrum of global geopotential field of Gaussian- bell type. Journal of the Korean Earth Science Society, 34, 393-401.

Dickinson, R.E., 1978, Rossby waves-long-period oscillations of oceans and atmospheres. Annual Review of Fluid Mechanics, 10, 159-195.

Dilts, G.A., 1985, Computation of spherical harmonic expansion coefficients via FFT's. Journal of Computational Physics, 57, 439-453.

Flyer, N., Lehto, E., Blaise, S., Wright, G., and St-Cyr, A., 2012, A guide to RBF-generated finite difference for nonlinear transport: Shallow water simulations on a sphere. Journal of Computational Physics, 231, 40784095.

Heikes, R.P. and Randall, D.A., 1995, Numerical integration of the shallow water equations on a twisted icosahedral grid. Part I: Basic design and results of tests. Monthly Weather Review, 123, 1862-1880.

Heikes, R.P., Randall, D.A., and Konor, C.S., 2013, Optimized icosahedral grids: Performance of finitedifference operators and multigrid solver. Monthly Weather Review, 141, 4450-4469.

Holton, J.R., 2004, An introduction to dynamic meteorology. Elsevier, California, USA, $522 \mathrm{p}$.

Jekeli, C., Lee, J.K., and Kwon, J.H., 2007, On the computation and approximation of ultra-high-degree spherical harmonic series. Journal of Geodesy, 81, 603615.

Moriguchi, S.I., Udakawa, K.H., and Shin, H.M., 1990, Formulas for mathematical functions III. The $5^{\text {th }}$ ed. Iwanami Shoten, Tokyo, $310 \mathrm{p}$.

Nair, R.D., 2009, Diffusion experiments with a global discontinuous Galerkin shallow-water model. Monthly Weather Review, 137, 3339-3350.

Nehrkorn, T., 1990, On the computation of Legendre functions in spectral models. Monthly Weather Review, 118, 2248-2251.

Rossby, C.G., 1939, Relation between variations in the intensity of the zonal circulation of the atmosphere and the displacements of the semi-permanent centers of action. Journal of Marine Research, 2, 38-55.

Sneeuw, N. and Bun, R., 1996, Global spherical harmonic computation by two-dimensional Fourier method. Journal of Geodesy, 70, 224-232.

Stuhne, G.R. and Peltier, W.R., 1995, Vortex erosion and amalgamation in a new model of large scale flow on the sphere. Journal of Computational Physics, 128, 5881.

Swarztrauber, P.N., 1993, The vector harmonic transform method for solving partial differential equations in spherical geometry. Monthly Weather Review, 121, 3415-3437.

Swarztrauber, P.N., 1996, Spectral transform methods for solving the shallow water equations on the sphere. 
Monthly Weather Review, 124, 730-744.

Thuburn, J., Ringler, T.D., Skamarock, W.C., and Klemp, J.B., 2009, Numerical representation of geostrophic modes on arbitrarily structured C-grids. Journal of
Computational Physics, 228, 8321-8335.

Weller, H., 2012, Controlling the computational modes of the arbitrary structured C-grid. Monthly Weather Review, $140,3220-3234$.

Manuscript received: July 24, 2014 Revised manuscript received: August 8, 2014 Manuscript accepted: August 17, 2014 